\title{
Assimilação dos Dados do Experimento de Copenhagen em um Modelo Advectivo-Difusivo Aplicado a Dispersão de Poluentes na Camada Limite Atmosférica
}

\author{
$\underline{\text { Simone M. Strieder }}{ }^{1 *}$ \\ Régis S. Quadros ${ }^{2}$
}

Fabrício P. Härter ${ }^{1}$

Daniela Buske ${ }^{2}$
${ }^{1}$ Depto de Meteorologia, FMET, UFPel,

${ }^{2}$ Depto de Matemática e Estatística, IFM, UFPel, 96010-610, Pelotas, RS

E-mail: simonestrieder@yahoo.com.br, quadros99@gmail.com, E-mail: fabricio.harter@ufpel.edu.br, danielabuske@gmail.com

\section{RESUMO}

A preservação da qualidade do ar é necessária para a boa manutenção da qualidade de vida no nosso planeta. A poluição, que pode ser causada por efeitos naturais e antropogênicos, é motivo de preocupação, principalmente nos grandes centros urbanos. Quanto as causas naturais da poluição, tais como erupções vulcânicas, responsáveis pela liberação de $\mathrm{SO}_{2}$, pouco ou nada pode-se fazer para reduzila. Todavia, a poluição antropogênica pode e deve ser controlada e/ou reduzida. Para tal, são feitos experimentos para medição de concentração de poluentes, tal como Copenhagen (1981) e previsões de dispersão de poluentes por modelos matemáticos.

Na Camada Limite Atmosférica (CLA) estima-se o campo de concentração de poluentes através da equação de advecção-difusão, (Zannetti, 1990). Estes modelos podem ser resolvidos por métodos numéricos e, alguns tem soluções analíticas ou híbridas (analítico-numéricas).

Entretanto, a precisão dos modelos numéricos diminuem com o horizonte de previsão, pois as condições inicias são imprecisas, há erros de truncamento em modelos em diferenças finitas e erros de arredondamento em modelos espectrais, entre outros. Por esta razão utiliza-se técnicas de assimilação de dados para a correção da trajetórias de modelos de dispersão de poluentes, previsão de tempo, modelos de previsão de correntes oceânicas, etc. O estado da arte em técnicas de assimilação de dados são técnicas baseados na filtragem de Kalman e métodos variacionais (Kalnay, 2004).

Métodos de assimilação de dados consistem em combinar dados observados com modelos numéricos com base no conhecimento estatístico dos erros de modelagem e de observação.

Neste trabalho, resolve-se a equação de advecção-difusão bidimensional para o caso estacionário, modelo de fechamento de primeira ordem para as equações dos fluxos turbulentos, com coeficiente de difusão turbulenta seguindo a modelagem de Degrazia et al (1997). Aplica-se a técnica de diferenças finitas, sendo o método de Euler explícito para integração na direção-x e diferenças centradas para discretização do operador de difusão (Hoffman, 1993) e assimila-se os dados do experimentos de Copenhagen através do filtro de Kalman Estendido (EKF) e do Método Variacional Tridimensional (3D-Var). O objetivo é corrigir a trajetória do modelo, diminuindo o erro de previsão.

Com o propósito de testar o esquema de assimilação, os seguintes parâmetros foram usados: $\Delta \mathrm{x}=$ $\mathbf{0}, \mathbf{5} \mathrm{m}, \Delta z=\mathbf{1 0} \mathrm{m}$, altura da camada limite $\mathbf{h}=\mathbf{8 1 0} \mathrm{m}$ e altura de fonte $\mathbf{h f}=\mathbf{1 1 5} \mathrm{m}$. No EKF a matriz que representa o sistema de observação é $\mathbf{H}=\mathbf{I}$, e as matrizes de covariância dos ruídos de

\footnotetext{
*bolsista de Iniciação Científica PBIP/CNPq
} 


\begin{tabular}{|c|c|c|c|}
\hline $\mathrm{X}$ & Modelo & EKF & 3D-Var \\
\hline $1.900 \mathrm{~m}$ & 0,5756 & 0,0727 & 0,0003 \\
$3.600 \mathrm{~m}$ & 0,6177 & 0,0798 & 0,0001 \\
$5.300 \mathrm{~m}$ & 0,9948 & 0,1245 & 0,0000 \\
\hline
\end{tabular}

Tabela 1: Erro Quadrático da previsão de concentração pelo modelo sem assimilação e com assimilação por EKF e 3D-Var ao nível do solo para 1.900, 3.600 e $5.300 \mathrm{~m}$ da fonte.

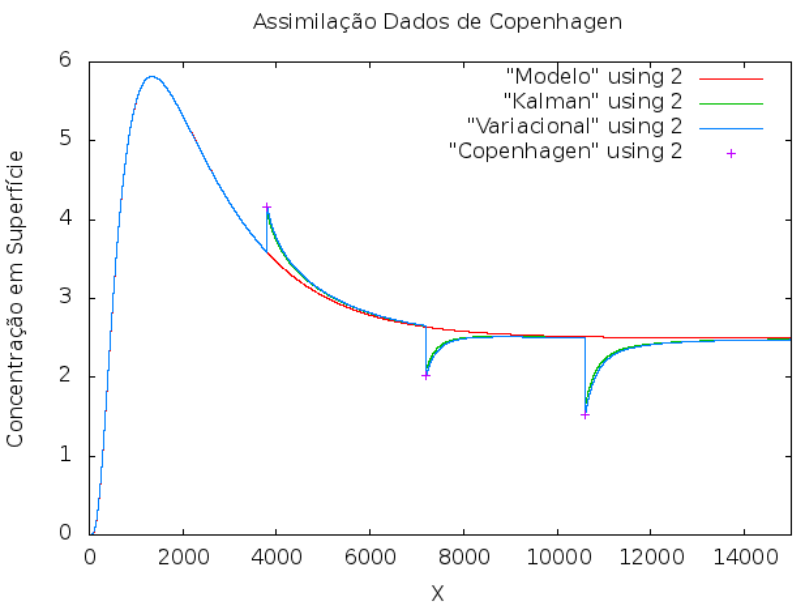

Tabela 2: Assimilação dos Dados de Copenhagen.

observação e modelagem, são respectivamente $\mathbf{R}_{\mathbf{n}}=\mathbf{2 I}$ e $\mathbf{Q}_{\mathbf{n}}=\mathbf{0}, \mathbf{5 I}$, sendo que $\mathbf{I}$ é a matriz identidade da ordem de $N_{z}=41$. No 3D-Var, a função custo foi minimizada com o gradiente desendente em 200 interações. O número de pontos na direção $x$ é $15.000(7.500 \mathrm{~m})$. Este problema de difusão simula uma pluma liberada na origem do sistema de coordenadas. O resultado é grafado na Tabela 2.

Os resultados apresentados na tabela e figura acima mostram que o procedimento de assimilação corrige a trajetória do modelo. Observa-se que o 3D-Var é quase ótimo para o experimento em questão, onde o erro de observação foi considerado muito pequeno. Em outros níveis verticais (não mostrados neste texto), a assimilação não tem efeito considerável, pois assimilou-se dados apenas em superfície e em três pontos bastante distantes. Segundo Härter et al (2002) os resultados melhoram proporcionalmente ao aumento da frequência de observação. Nos experimentos grafados, observa-se que a poucos metros do ponto de observação, a assimlação perde efeito.

Palavras-chave: Dispersão de Poluentes, Assimilação de Dados, Filtro de Kalman, Experimento de Copenhagen

\section{Referências}

[1] S. E. Gryning, 1981, Elevated source SF 6 - tracer dispersion experiments in the Copenhagen area. Report RISOE-R-446, Riso National Laboratory, Roskilde, Denmark.

[2] P. Zannetti, “Air Pollution Modelling”, Comp. Mech. Publications, Southampton, 1990.

[3] E. Kalnay, "Atmospheric Modelling, Data Assimilation and Predictability", Cambridge University Press, 2003.

[4] F. P. Härter, Kalman Filtering in the Air Quality Monitoring, Ciência e Natura, (2002) 177-187.

[5] J. D. Hoffman, "Numerical methods for engineers and scientists", McGraw-Hill, Inc., 1993.

[6] G. A. Degrazia, H. F. Campos Velho e J. C. Carvalho, Nonlocal Exchange Coefficients for the Convective Boundary Layer Derived from Spectral Properties, Contributions to Atmospheric Physics, 1997, 57-64. 